\title{
The effect of different mechanisms of myocardial ischemia on left ventricular function
}

\author{
Robert E. Carlson, BS, Kevin M. Kavanaugh, MD, and Andrew J. Buda, MD. \\ Ann Arbor, Mich.
}

Myocardial ischemia occurs as a result of an imbalance between myocardial oxygen supply and demand. Therefore ischemia can be produced either by a decrease in coronary blood flow, which may be termed "supply-type" ischemia, or alternatively by an increase in myocardial oxygen demand in the setting of a fixed coronary stenosis, which may be termed "demand-type" ischemia. The clinical correlate of supply-type ischemia is variant angina, which is associated with coronary artery spasm, whereas demand-type ischemia is typical of the classic exertional angina associated with artherosclerotic coronary artery disease. However, regardless of the mechanisms that produce ischemia, myocardial dysfunction occurs within seconds of its onset. ${ }^{1}$

Two recent studies ${ }^{2,3}$ have characterized both the local functional and metabolic abnormalities of supply-type and demand-type ischemia, but there has been no report to date that has directly compared the spatial extent and severity of these two types of ischemic dysfunction. To address this issue, we performed circumferential left ventricular analyses of both regional tissue blood flow and functional parameters in dogs by means of two different models of ischemia. Supply-type ischemia was induced by abrupt circumflex coronary artery occlusion. Demand-type ischemia was produced by increasing myocardial oxygen demand pharmacologically with dobutamine, a potent inotropic agent, after the

From the Cardiology Division, Department of Internal Medicine, University of Michigan Medical School.

Supported in part by a grant-in-aid from the American Heart Association of Michigan, Lathrup Village, Mich.; grant HL 34691 from the National Heart, Lung, and Blood Institute, Bethesda, Md.; a Research Fellowship from the American Heart Association of Michigan (Dr. Kavanaugh); and a Student Research Fellowship from the American Heart Association of Michigan (Mr. Carlson).

Received for publication Dec. 14. 1987: acrented A pril 15, 1980.

Reprint requests: Andrew J. Buda, MD, Cardiology Division, University of Michigan Medical Center, 1500 E. Medical Center Dr., TC-3910. Ann Arbor, MI 48109-0366. creation of a single-vessel critical coronary stenosis.

\section{METHODOLOGY}

A total of 13 anesthetized, open-chest, male mongrel dogs (16 to $23 \mathrm{~kg}$ ) were studied. Each animal was anesthetized intravenously with thiamylal (20 $\mathrm{mg} / \mathrm{kg})$, followed by pentobarbital $(30 \mathrm{mg} / \mathrm{kg})$ as needed, and underwent a left-sided thoracotomy in the fifth intercostal space. Catheters were placed into the left internal jugular vein for administration of fluids and drugs, the left carotid and left and right femoral arteries for withdrawal of reference arterial blood samples, and the left atrial appendage for administration of radioactive-labeled microspheres. A proximal portion of the left circumflex coronary artery $(\mathrm{LCx})$ was isolated, and an electromagnetic flow probe was placed around the vessel with a snare-type occluder placed just distal to the probe. For the stenosis group, a screw-type occluder was placed on the LCx distal to the flow probe and proximal to the snare. Both groups received brief 15-second occlusions to zero the flow probe and produce a hyperemic response. A critical coronary stenosis was produced by adjusting the screw occluder until the peak hyperemic response was abolished without a change in baseline blood flow.

Experimental protocol. After preparation of the animal models, baseline short-axis, two-dimensional echocardiography was performed at the level of the midpapillary muscle. Concurrently, baseline hemodynamic measurements were obtained and microsphere injections were performed. After this baseline period, the supply-type ischemia group underwent LCx coronary occlusion. After approximately 10 minutes of occlusion, these animals received a second microsphere infusion and repeat two-dimensional echocardiography was performed. To induce ischemia in the group with demand-type ischemia, intravenous dobutamine $(15 \mu \mathrm{g} / \mathrm{kg} / \mathrm{min})$ was admin- 


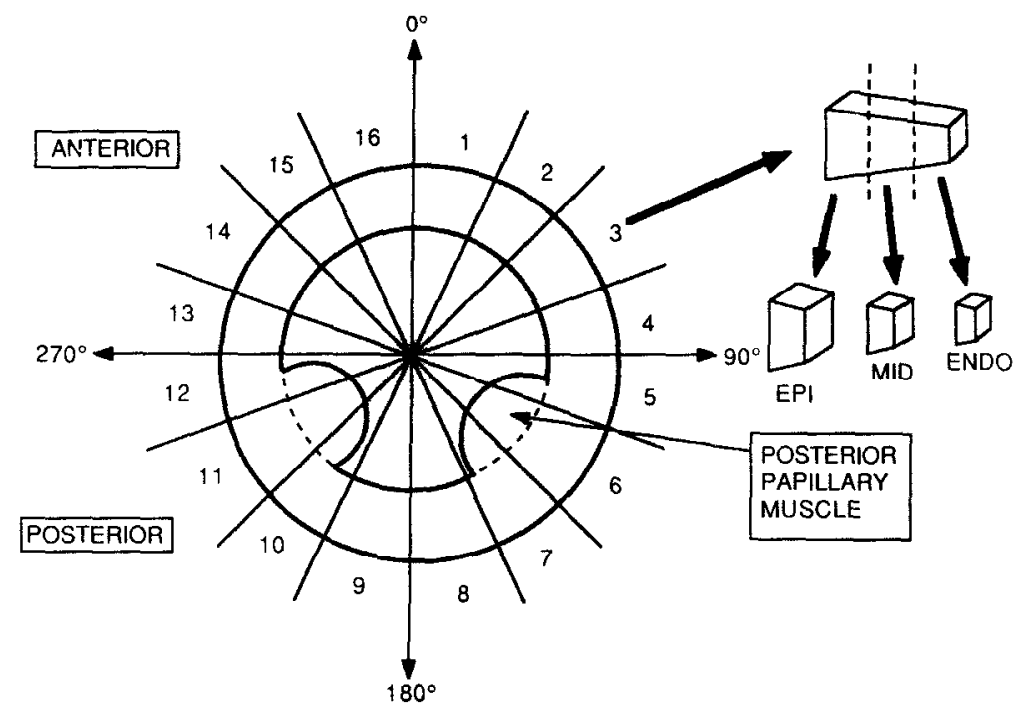

Fig. 1. Schematic illustration of method of circumferential blood flow analysis. Short-axis slice of left ventricle at level of midpapillary muscle was divided into 16 segments; each segment was then cut into subepicardial ( $E P I)$, midmyocardial (MID), and subendocardial (ENDO) pieces for analysis of radioactive microsphere content. Two-dimensional echocardiograms were analyzed by means of similar radial divisions.

istered to these animals for 10 minutes; identical flow and function measurements were then obtained.

Regional myocardial blood flow determination. The distribution of regional coronary blood flow was determined by the reference withdrawal technique using methods previously described. ${ }^{4}$ For each determination approximately 1 to $2 \times 10^{6}$ carbonized plastic microspheres ( $15 \mu \mathrm{m}$ diameter), labeled with $\mathrm{Sc}^{46}, \mathrm{Ru}^{103}$, or $\mathrm{Ce}^{141}$, were infused. At completion of the experiment, animals were killed by injection of potassium chloride, hearts were excised, and the left ventricle was isolated. Two $5 \mathrm{~mm}$ slices of the left ventricle corresponding to the level of the midposterior papillary muscle were obtained and dissected into sixteen 22.5-degree full-thickness sectors corresponding to the appropriate sectors obtained through the two-dimensional echocardiographic regional wall thickening data (Fig. 1). Each section was then cut into three pieces of approximately equal thickness from endocardium to epicardium. Each piece of tissue was weighed and placed in a gamma scintillation counter. After correcting for background and overlapping counts through computer analysis, tissue blood flow was calculated with the equation $Q_{m} / C_{m}=Q_{r} / C_{r}$, where $Q_{m}$ and $Q_{r}$ are the respective myocardial blood flow $(\mathrm{ml} / \mathrm{min})$ and withdrawal rate of the reference arterial blood sample $(\mathrm{ml} / \mathrm{min})$, and $C_{m}$ and $C_{r}$ are the respective counts per minute in the tissue sample and counts per minute in the reference arterial sample. Flow per gram of tissue was calculated by dividing flow by the weight of the appropriate sample. Both central ischemic and nonischemic regions of tissue blood flow were obtained by graphically representing the tissue blood flow $(\mathrm{ml} / \mathrm{min} / \mathrm{gm})$ for the baseline and ischemia time points by means of circumferential blood flow maps.

Echocardiographic analysis. With the use of a Diasonics CRC minicomputer-based video digitizing system (Diasonics, Milpitas, Calif.). end-diastolic and end-systolic frames were selected for analysis by means of the onset of the $Q$ wave in lead II to define end diastole and the smallest ventricular cavity to define end systole. An experienced observer, who was unaware of the experimental protocol, carefully traced endocardial and epicardial borders directly from the video display for three successive heart beats by means of a digitizing tablet. Analysis of regional wall thickening was performed by means of a radial contraction model with a fixed diastolic center of mass at 22.5-degree intervals over the full left ventricular circumference. For correction of rotation, the midpoint of the posterior papillary muscle was fixed at 135 degrees. Wall thickening was calculated as: (end-systolic wall thickness - end-diastolic wall thickness/end-diastolic wall thickness $\times 100 \%$. 

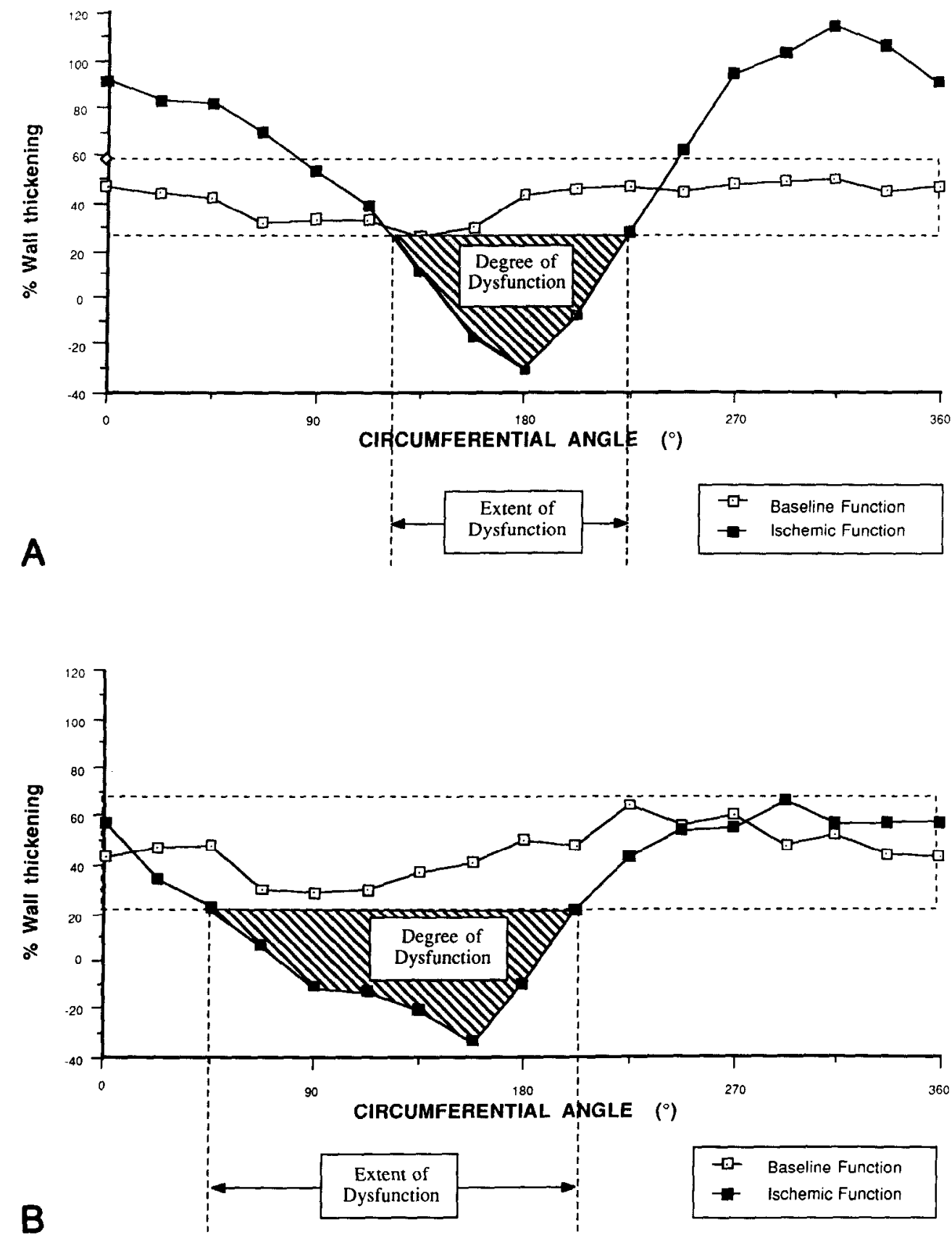

Fig. 2. $A$, Map of circumferential left ventricular function during demand-type ischemia. $B$, Map of circumferential function during supply-type ischemia. Note differences in severity of ischemic functional responses. Extent of dysfunction as determined by circumferential portion of left ventricle, which functions below baseline limits, is larger for supply-type ischemia compared to demand-type ischemia. Similarly, degree of dysfunction is larger for supply-type ischemia and suggests that ischemic tissue segments are more dyskinetic as a result of coronary occlusion compared to pharmacologically induced increased myocardial oxygen demand in presence of coronary stenosis.

For each time point, the mean \pm standard deviation was calculated for three normal beats for which 95\% tolerance intervals were established from the baseline wall thickening values. The wall thickening values at baseline were then compared to those valnes during ischemia and superimposed onto a map that circumferentially expressed these data in terms of the regions of the left ventricle being examined. Abnormally functioning regions that were below the functional tolerance limits set by the 

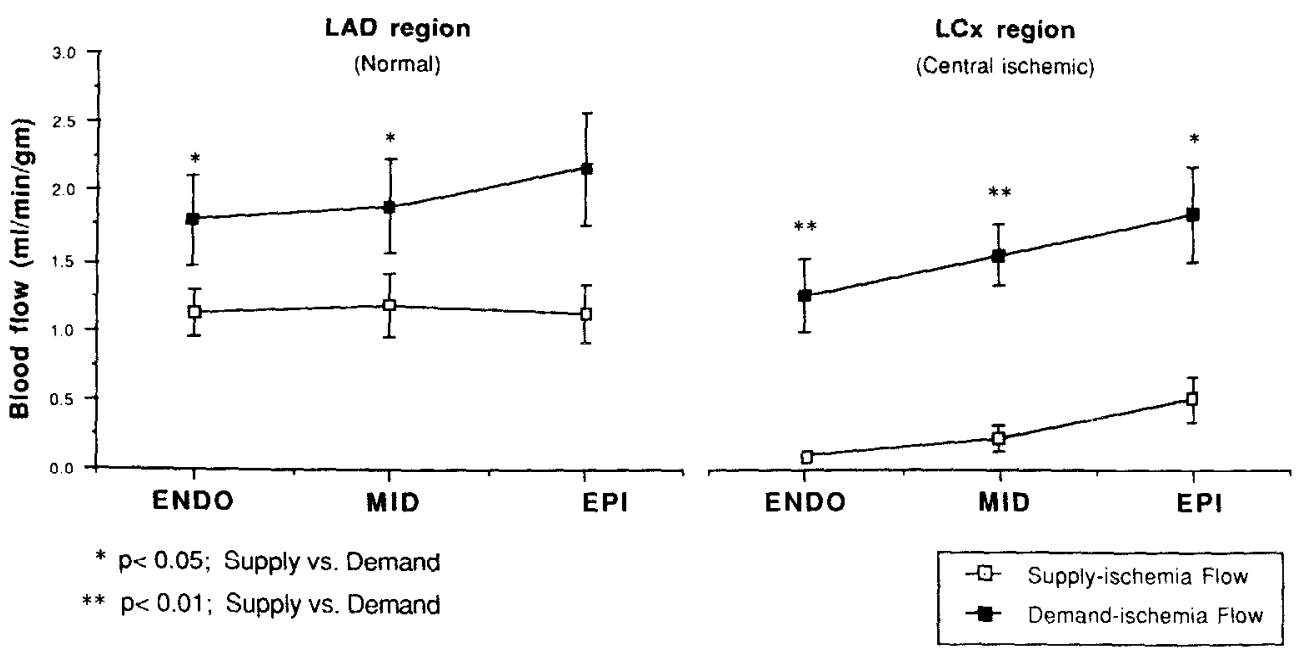

Fig. 3. Blood flow in three myocardial tissue levels during ischemia is compared in LAD and LCx regions of left ventricle. As a result of particular ischemia-producing maneuver, differences in myocardial blood flow in subendocardial (ENDO), midmyocardial (MID), and epicardial (EPI) layers are apparent. Demand-type ischemia resulted in higher levels of flow relative to supply-type ischemia.

Table I. Hemodynamic and left ventricular global parameters

\begin{tabular}{lcccc}
\hline & \multicolumn{2}{c}{ Supply } & \multicolumn{2}{c}{ Demand } \\
\cline { 2 - 5 } & Baseline & Ischemia & Baseline & Ischemia \\
\hline HR (beats/min) & $149 \pm 6$ & $147 \pm 7$ & $144 \pm 6$ & $157 \pm 8^{*}$ \\
MAP (mm Hg) & $124 \pm 4$ & $113 \pm 4^{*}$ & $109 \pm 2$ & $155 \pm 16^{*}$ \\
LVED area $\left(\mathrm{cm}^{2}\right)$ & $12.51 \pm 0.70$ & $14.91 \pm 0.63^{*}$ & $9.65 \pm 0.84 \ddagger$ & $9.75 \pm 1.12 \dagger$ \\
LVES area $\left(\mathrm{cm}^{2}\right)$ & $6.05 \pm 0.59$ & $10.20 \pm 0.77^{* *}$ & $4.02 \pm 0.51 \ddagger$ & $3.76 \pm 0.84 \dagger$ \\
LV area EF $(\%)$ & $0.52 \pm 0.02$ & $0.32 \pm 0.03^{* *}$ & $0.59 \pm 0.02$ & $0.63 \pm 0.05 \dagger$ \\
\hline
\end{tabular}

$\mathrm{EF}=$ Ejection fraction; $\mathrm{HR}=$ heart rate; $\mathrm{MAP}=$ mean arterial pressure $; \mathrm{LVED}=$ left ventricular end-diastolic; $\mathrm{LVES}=$ left ventricular end-systolic. ${ }^{*} p<0.05$ vs baseline.

${ }^{* *} p<0.01$ vs baseline.

$t_{p}<0.01$; supply vs demand.

$\ddagger p<0.05$; supply vs demand.

baseline values were determined from the functional maps and expressed as the circumferential extent and degree of dysfunction (area units). The extent of dysfunction was defined as the number of degrees between curve intercepts of the lower tolerance limit for the circumferential map of the ischemic and baseline wall thickening data (Fig. 2, $A$ and $B$ ). The degree of dysfunction was measured as the planimetered area of the map that was enclosed by the baseline lower tolerance limit and the dysfunctional portion of the ischemic wall thickening curve. (Fig. $2, A$ and $B$ ).

Statistical analysis. All data are expressed as mean \pm standard error of the mean. Appropriate statistical analysis of values was made by means of Student's and Behrens-Fischer ( $F$ value $>4$ ) unpaired and paired $t$ tests as appropriate. The probability $(p)$ was considered to be statistically significant when $p<0.05$.

\section{OBSERVATIONS}

Hemodynamic parameters. The hemodynamic and left ventricular global functional data are summarized in Table I. The group with supply type ischemia had no significant changes in heart rate and a decrease in mean arterial pressure during the ischemic insult compared to baseline values $(p<0.05)$. The group with demand type ischemia had significant increases in both heart rate $(p<0.05)$ and blood pressure $(p<0.05)$ during this period. An analysis of left ventricular cavity areas as determined by two-dimensional echocardiography showed baseline differences in both left ventricular end-diastolic and end-systolic area measurements. 
Table II. Regional myocardial blood flow

\begin{tabular}{|c|c|c|c|c|}
\hline \multirow[b]{2}{*}{ Region } & \multicolumn{2}{|c|}{$L A D$} & \multicolumn{2}{|c|}{$L C x$} \\
\hline & Supply & Demand & Supply & Demand \\
\hline \multicolumn{5}{|c|}{ Subendocardium } \\
\hline Baseline & $1.05 \pm 0.14$ & $1.09 \pm 0.16$ & $1.22 \pm 0.23$ & $1.08 \pm 0.11$ \\
\hline Ischemia & $1.13 \pm 0.17$ & $2.03 \pm 0.33^{* *}, \ddagger$ & $0.09 \pm 0.04^{* *}$ & $1.04 \pm 0.07 \dagger$ \\
\hline \multicolumn{5}{|c|}{ Midmyocardium } \\
\hline Baseline & $0.98 \pm 0.12$ & $1.02 \pm 0.11$ & $1.07 \pm 0.12$ & $0.96 \pm 0.07$ \\
\hline Ischemia & $1.18 \pm 0.23$ & $1.90 \pm 0.33^{*}, \ddagger$ & $0.23 \pm 0.09^{* *}$ & $1.56 \pm 0.22^{*}, \dagger$ \\
\hline \multicolumn{5}{|c|}{ Subepicardium } \\
\hline Baseline & $1.09 \pm 0.21$ & $1.04 \pm 0.13$ & $1.10 \pm 0.24$ & $1.03 \pm 0.08$ \\
\hline Ischemia & $1.12 \pm 0.21$ & $2.17 \pm 0.41^{* *}$ & $0.51 \pm 0.17^{*}$ & $1.85 \pm 0.34^{*}, \dagger$ \\
\hline
\end{tabular}

All values are in $\mathrm{ml} / \mathrm{min} / \mathrm{gm}$.

${ }^{*} p<0.05$ vs baseline.

${ }^{* *} p<0.01$ vs baseline.

$\uparrow p<0.01$; supply vs demand.

$\ddagger p<0.05$; supply vs demand.

Table III. Regional wall thickening

\begin{tabular}{llccc} 
& & & \multicolumn{2}{c}{$L C x$} \\
\cline { 3 - 5 } & Supply & Demand & Supply & Demand \\
\hline Baseline & $39.5 \pm 2.2$ & $45.3 \pm 1.3$ & $33.6 \pm 2.2$ & $38.8 \pm 2.5$ \\
Ischemia & $63.6 \pm 7.6^{*}$ & $85.2 \pm 7.1^{* *}$ & $-16.2 \pm 3.7^{* *}$ & $0.87 \pm 5.9^{* *}, \ddagger$ \\
\hline
\end{tabular}

All values are percentages of wall thickness.

${ }^{* *} p<0.01$ vs baseline.

$\ddagger p<0.05$; supply vs demand.

Although these differences in baseline cavity area were present, a comparison of the left ventricular area ejection fractions resulting from these values showed no difference. However, during ischemia the group with supply-type ischemia had significant increases in left ventricular end-diastolic and endsystolic areas and a significant decrease in the left ventricular area ejection fraction compared to baseline values $(p<0.01)$. The ischemic condition generated in the demand model did not produce a change in area ejection fraction, suggesting that the increased function in the nonischemic myocardium produced by dobutamine offset any global dysfunction. A resulting intergroup comparison of left ventricular cavity areas indicated that supply-type ischemia caused both end-systolic and end-diastolic areas to increase significantly relative to the demand-type ischemia. This resulted in a significantly lower mean left ventricular area ejection fraction for the group with supply type ischemia cuispareu iv ine group with demand type ischemia $(p<0.01)$.

Myocardial blood flow. Baseline and ischemic myocardial blood flow values for subepicardial, midmyo- cardial, and subendocardial segments in both the nonischemic left anterior descending (LAD) region and the ischemic LCx region are summarized in Table II. An intergroup comparison of baseline blood flow values for the three layers of the myocardium showed no significant differences for associated segments in either the LAD or LCx regions of the left ventricle. However, with the onset of ischemiainduced left ventricular dysfunction, an intergroup comparison of the LCx regions (Fig. 3) indicated a marked difference in subendocardial blood flow between the groups with supply-type and demandtype ischemia $(0.09 \pm 0.04$ vs $1.04 \pm 0.07 \mathrm{ml} / \mathrm{min} /$ gm; $p<0.01$ ). This difference was also apparent at the midmyocardial and subepicardial ischemic tissue levels (Fig. 3). Compared to their respective baseline values for subendocardial blood flow, dissimilar changes occurred between the groups in relation to ischemic blood flow; the group with supply-type ischemia had a significant decrease in LCx tissue blood flow, whereas in the group with demand-type ischemia LCx blood flow did not change from baseline values (Table II). For both the midmyocardial and subepicardial layers, LCx tissue 


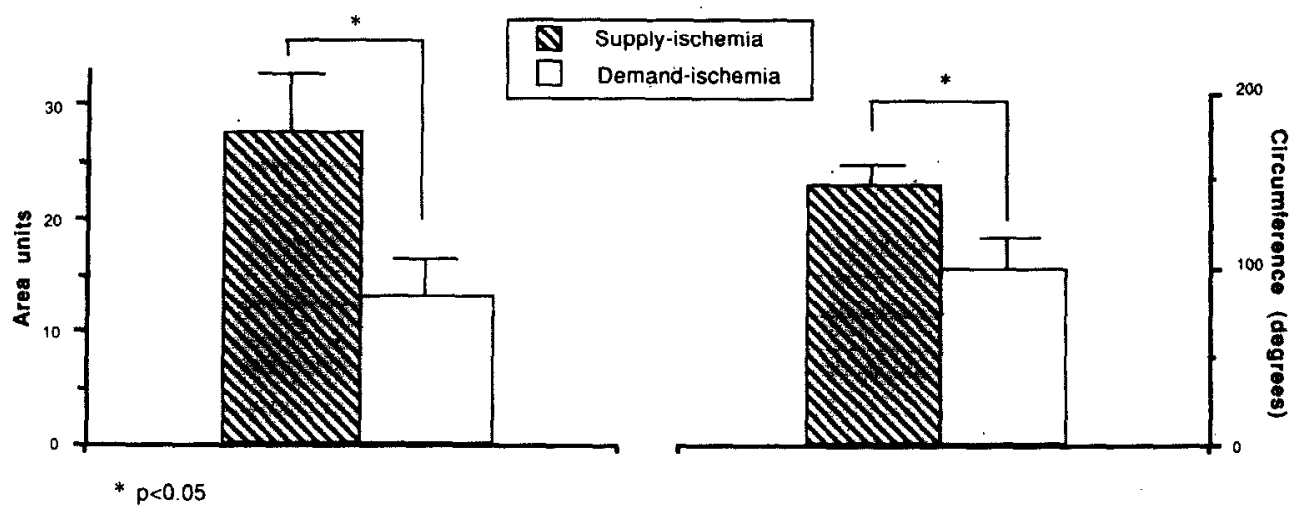

Fig. 4. Intergroup comparisons of both degree and extent of left ventricular dysfunction, reflecting relative severity of supply-type and demand-type ischemia. Note group with supply-type ischemia had both a larger degree of dysfunction and extent of dysfunction compared to similar measures of dysfunction produced by demand-type ischemia.

blood flow increased significantly in the group with demand-type ischemia, whereas severe reduction below baseline values was present in the group with supply-type ischemia.

Blood flow to the nonischemic, subendocardial LAD region during the ischemic episode was lower (1.13 $\pm 0.17 \mathrm{ml} / \mathrm{min} / \mathrm{gm}$ ) for the group with supplytype ischemia compared to the group with demandtype ischemia $(2.03 \pm 0.33 \mathrm{ml} / \mathrm{min} / \mathrm{gm} ; p<0.05)$. An intragroup comparison indicated that $L A D$ tissue blood flow was augmented at all three myocardial layers for both groups relative to their baseline flow values; the group with supply-type ischemia showed trends, whereas the group with demandtype ischemia had significant increases in blood flow, reflecting the increased myocardial oxygen demands produced by infusion of dobutamine in this latter group (Table II).

Regional loft ventricular function relations. An analysis of the functional parameters obtained by twodimensional echocardiography indicated that significant differences exist between the ischemia produced by occlusion and that produced by stenosis and increased myocardial oxygen demands (Table III). With the onset of ischemia, the occluded animals had immediate left ventricular dysfunction characterized by enlargement of the myocardial cavity and ECG evidence of ST segment elevations, whereas more variable yet similar responses were observed in the group with demand-type ischemia. Examples of circumferential function maps comparing the extent and degree of dysfunction for the supply-and demand-types of ischemia are shown in Fig. 2, $A$ and $B$. No significant differences were found when baseline percentages of wall thickening for the LAD and LCx regions were compared between the groups with supply-type and demandtype ischemia (Table III). During the ischemic period, the percentage of wall thickening in the LCx region was significantly different for the group with supply-type ischemia $(-16.2 \pm 3.7 \%)$ compared to the group with demand-type ischemia $(0.9 \pm 5.9 \%$; $p<0.05$ ). After generation of circumferential wall thickening maps comparing baseline and ischemia time points, the extent of dysfunction for the group with supply-type ischemia was greater compared to the group with demand-type ischemia $(p<0.05$; Fig. 4). Similarly, the degree of dysfunction was greater in the group with supply-type ischemia $(p<0.05$; Fig. 4).

\section{COMMENTS}

Our results clearly indicate that supply-type ischemia produces a greater extent and degree of left ventricular dysfunction than demand-type ischemia. Despite the occurrence of ischemia-related dysfunction in both experimental conditions, the difference in the resulting left ventricular dysfunction likely reflects differences in the severity of the particular ischemic stress. This is supported by the fact that coronary occlusion produced a marked decrease in myocardial blood flow in all layers of the myocardium; whereas pharmacologic stress-induced ischemia was associated with less severe reductions in myocardial blood flow and a relative redistribution of blood flow from the subendocardium to the subepicardium. These distinct differences in patterns of ischemic myocardial blood flow largely help to explain the heterogeneous functional consequences of these two distinct ischemic insults. 
Changes in myocardial blood flow after complete coronary occlusion have been previously well investigated..$^{6-9}$ As demonstrated in our study, at the time of occlusion, a marked and severe reduction in blood flow occurs in all three layers of the myocardium and is particularly marked in the subendocardial layer. This is important because regional contractile function is almost linearly related to subendocardial blood flow, and thus incremental decreases in subendocardial blood flow are associated with a proportional decrease in regional myocardial wall thickness. This close coupling of subendocardial blood flow and regional left ventricular function was initially explored by means of unidimensional techniques $^{7-9}$ such as implanted sonomicrometers, but more recently two-dimensional echocardiography has been used..$^{5,10}$ Whereas sonomicrometry provides highly accurate and precise data on regional function, two-dimensional echocardiography characterizes the spatial distribution of the regional functional abnormality. In this sense, the two techniques are highly complimentary in comprehensively characterizing the functional consequences of acute myocardial ischemia.

The extent and degree of left ventricular dysfunction after acute coronary occlusion that was found by two-dimensional echocardiography in this study are consistent with findings in our previous studies ${ }^{5,11,12}$ and those of others. ${ }^{13-16}$ However, only a few studies have investigated tissue blood flow and changes in myocardial function after increased cardiac stress in the setting of a critical stenosis, ${ }^{17-19}$ and only two previous studies ${ }^{2,3}$ have specifically compared the functional consequences of supply-type and demand-type ischemia. In one recent study by Paulus et al., ${ }^{2}$ myocardial function was measured by pressure-segment length relations after pacing tachycardia in dogs with coronary stenoses and was compared to function of the same segment during coronary occlusion. In their study, ischemia produced by coronary stenoses plus a tachycardiainduced increase in myocardial oxygen demands (demand-type ischemia) had substantially different effects on regional wall motion and segmental diastolic mechanics from the ischemia produced by coronary occlusion. In association with this demand-type ischemia, diastolic dysfunction was a major characteristic as indicated by an upward and rightward shift of the diastolic pressure-segment length relation and prolongation of the time consiani (iau) of left ventricular pressure decay. With ischemia resulting from coronary occlusion (supplytype ischemia), systolic dysfunction was the major feature and similar analysis of diastolic dysfunction was minimal. However, no attempt was made in their study to measure the extent of the observed ischemic abnormalities. Thus, although diastolic function was not assessed in our study, the present observations agree with and further extend the results of Paulus et al. ${ }^{2}$ to indicate that the spatial distribution of the functional abnormality measured with these two different forms of ischemia also differs substantially.

In addition to rapid pacing, other interventions have been used to increase myocardial oxygen demands in the presence of a coronary artery stenosis, including physiologic exercise ${ }^{17,18}$ or administration of sympathomimetic drugs. ${ }^{19,20}$ In our study, dobutamine infusion was used in animals with a single-vessel critical stenosis. Although this pharmacologic stress intervention may not produce the most rigorous cardiovascular stress compared to other interventions, dobutamine did significantly increase the heart rate-blood pressure double product and in this way simulated the physiologic exercise state. Furthermore, we chose dobutamine as a stress intervention because it is being used clinically with increasing frequency in combination with a variety of cardiac imaging approaches as an alternative stress procedure. ${ }^{22,23}$

Dobutamine did not change the mean transmural myocardial blood flow but produced a redistribution of blood flow from the subendocardium to the subepicardium and produced a concomitant decrease in the endocardial/epicardial ratio consistent with ischemia. This is in agreement with the experience of Gallagher et al., ${ }^{19}$ who used isoproterenol to induce cardiac stress. In their study, during infusion of isoproterenol with coronary stenosis, wall thickening was significantly decreased, although blood flow was comparable to conditions at rest, suggesting that the demand for blood flow exceeded the supply. Function began to decrease during isoproterenol-induced ischemia when subendocardial blood flow decreased to less than $150 \%$ to $200 \%$ of the resting level. In addition, reductions in wall thickening during infusion of isoproterenol correlated well with decreases in regional subendocardial blood flow. Also, in agreement with the findings of Gallagher et al ${ }^{10}$ during ischemia, there was a redistribution of blood flow from the subendocardium to the subepicardium. The augmentation in subepicardial flow most likely reflects decreased vascular resistance in the outer myocardium. In the setting of coronary stenosis, the reduced subepicardial resistance may have produced a transmural steal effect in which coronary inflow, limited in volume by the stenosis, preferentially perfused the outer myocardium at the expense of perfusion of the deeper layers. ${ }^{17}$ This redistribution of myocardial blood flow is 
particularly important in view of the major contribution of the subendocardium to overall transmural function both at rest and after inotropic stimulation. ${ }^{24}$ In the setting of inotropic challenge as produced with dobutamine, the contribution of subendocardial wall thickening in response to this increased myocardial oxygen demand is augmented, so that even a minor decrease in subendocardial perfusion may lead to ischemic dysfunction. This may explain the apparent paradox of left ventricular dysfunction despite seemingly adequate myocardial perfusion.

It is also interesting to note that compensatory augmentation of left ventricular function in the nonischemic LAD area occurred in both groups of animals after induction of myocardial ischemia. This hyperfunction in the nonischemic zones appears to be related to a local Frank-Starling phenomenon produced by an increased stretching of the myocardial segments in this area, related to the increased load caused by the dyskinetic segments of the ischemic myocardium..$^{25}$ Another probable mechanism may result from regional intraventricular unloading from the nonischemic area into the more compliant ischemic area producing this compensatory hypercontractile state. ${ }^{25}$ In addition, the animals that received the infusion of dobutamine had an even greater increase in nonischemic hyperfunction, which was most likely related to the synergistic effect of this inotropic agent.

There are a number of metabolic alterations between supply-type and demand-type ischemia that may have contributed to the functional results we observed. Complete coronary occlusion is known to be associated with a local increase of $[\mathrm{H}+]$ in the ischemic myocardium, and this has been shown to be more severe in supply-type ischemia than demandtype ischemia. ${ }^{26}$ The restricted but continued myocardial perfusion associated with demand-type ischemia is believed to limit local increases of $[\mathrm{H}+]$, thus acting to buffer changes in tissue $\mathrm{pH} .{ }^{3}$ Similarly, local stores of phosphocreatine, a precursor of high-energy phosphates, decrease significantly with supply-type ischemia of a complete coronary occlusion, whereas only minor decreases occur for this metabolite in demand-type ischemia. ${ }^{3}$ Furthermore, Momomura et al. ${ }^{3}$ found that greater depression of myocardial cell $\mathrm{Po}_{2}$ and high-energy phosphates was present during supply-type compared to demandtype ischemia. These disparate metabolic results further reflect the differences in the magnitude of severity of the ischemia produced and help explain the heterogeneity of our functional results.

There are alternative explanations for the differences in the functional abnormalities produced in the two groups of animals. It is possible that differences existed in the size of the myocardial area at risk rendered ischemic. Because we did not specifically measure areas at risk in this study, we cannot totally exclude this possibility. However, this appears unlikely because the same coronary artery, namely, the LCx, and the same anatomic site of coronary manipulation were selected in both groups of dogs. Although there may be individual variability of areas at risk, in our previous experience of infarct studies, using a similar site of occlusion on the $\mathrm{LCx},{ }^{12,27}$ the myocardial area at risk was similar in different groups of animals investigated. For both of these reasons, it is unlikely that differences in myocardial areas at risk explain the disparities in functional results between supply-type and demand-type ischemia. It is also possible that different severities of ischemia were produced; given the significant differences in myocardial blood flow responses, this seems likely. However, to absolutely determine whether this was indeed the case, a metabolic marker of ischemia would be necessary.

There are some important clinical implications of our observations. Supply-type ischemia is typical of variant angina resulting from transient coronary artery spasm or transient thrombotic occlusion. Demand-type ischemia is commonly observed as exertional angina associated with artherosclerotic coronary artery disease. Our findings suggest that coronary spasm or brief occlusion will produce greater functional abnormality than exertional angina. The marked functional abnormality observed by two-dimensional echocardiography in patients with variant angin $a^{28}$ and the marked hemodynamic deterioration that occurs in some of these patients during ischemia ${ }^{29}$ would tend to support this hypothesis. However, it should be emphasized that the clinical situation is much more complex than the experimental models we studied, because many patients have multivessel coronary involvement, mixed angina (both supply-type and demand-type ischemia), and coronary collaterals, all of which may modify the functional response. Nevertheless, our experimental findings suggest that functional deterioration in the clinical setting may be associated with both the type and severity of the ischemia produced.

In conclusion, our results indicate that the extent and degree of left ventricular dysfunction differ with the mechanisms of production of myocardial ischemia. Supply-type ischemia, which occurs after coronary artery occlusion, produces a greater extent and degree of left ventricular dysfunction compared to demand-type ischemia, which results from increased myocardial oxygen demands in the setting of coro- 
nary stenosis. These observations provide additional insight into the heterogeneous functional consequences of acute myocardial ischemia in humans.

\section{SUMMARY}

Myocardial ischemia may be produced by limitation of blood flow as in abrupt coronary occlusion, termed supply-type ischemia, or by increasing myocardial oxygen demand in the setting of restricted flow, termed demand-type ischemia. To examine the comparative extent and severity of the dysfunction related to both forms of ischemia, we studied anesthetized, open-chest dogs by means of two-dimensional echocardiography and tracer microspheres. Supply-type ischemia was produced by total occlusion of the LCx $(n=7)$; demand-type ischemia was induced by infusion of dobutamine after creation of a critical $L C x$ stenosis $(n=6)$. At the time of the production of ischemia, the group with demand-type ischemia had significant increases in both heart rate $(p<0.05)$ and mean arterial pressure $(p<0.05)$, whereas the group with supply-type ischemia had a decrease in mean arterial pressure $(p<0.05)$. Subendocardial blood flow in the $\mathrm{LCx}$ region was severely depressed in supply-type ischemia $(0.09 \pm$ $0.04 \mathrm{ml} / \mathrm{min} / \mathrm{gm}$ ) compared to demand-type ischemia $(1.04 \pm 0.07 \mathrm{ml} / \mathrm{min} / \mathrm{gm} ; p<0.01)$. Although both groups of animals had an abnormality of left ventricular function during ischemia, as determined by two-dimensional echocardiography, the extent of the dysfunction in the group with supply-type ischemia was greater (146 \pm 12 degrees) compared to the group with demand-type ischemia (99 \pm 9 degrees; $p<0.01$ ). Similarly, the degree of left ventricular dysfunction in the group with supply-type ischemia was greater than that for the group with demandtype ischemia $(p<0.05)$. Thus these data suggest that supply-type ischemia produced by coronary occlusion results in a greater extent and degree of left ventricular functional abnormality than pharmacologically induced demand-type ischemia.

We thank Diane P. Pace, BS, and Lisa Krause, MA, for excellent technical assistance.

\section{REFERENCES}

1. Tennant R, Wiggers CJ. The effect of coronary occlusion on myocardial contractions. Am .I Physiol 1935;112:351.

2. Paulus WJ, Grossman W, Serizawa T, Boudillon PD, Pasipoularides A, Mirsky I. Different effects of two types of ischemia on myocardial systolic and diastolic function. Am J Phvsiol 1985:248:H719

3. Momomura S, Ingwall JS, Parker JA, Sahagian P, Ferguson JJ, Grossman W. The relationships of high energy phosphates, tissue $\mathrm{pH}$, and regional blood flow to diastolic distensibility in the ischemic dog myocardium. Circ Res $1985 ; 57: 822$.
4. Heymann MA, Payne BD, Hoffman JIE, Rudolph AM. Blood flow measurements with radionuclide-labelled particles. Prog Cardiovasc Dis 1977;20:55.

5. Buda AJ, Zotz RJ, Gallagher KP. Characterization of the functional border zone around regionally ischemic myocardium using circumferential flow-function maps. J Am Coll Cardiol 1986;8:150.

6. Kerber RE, Marcus ML, Ehrhardt J, Wilson R, Abboud FM. Correlation between echocardiographically demonstrated segmental dyskinesis and regional myocardial perfusion. Circulation 1975;52:1097.

7. Stowe DF, Mathey DF, Moores WY, Glantz SA, Townsend RM, Kabra P, Chaterjee K, Parmley WW, Tyberg JV. Segmental stroke work and metabolism depend on coronary blood flow in the pig. Am J Physiol 1978;234:H597.

8. Gallagher KP, Kumada T, Koziol JA, McKown W, Kemper WS, Ross Jr J. Significance of regional wall thickening abnormalities relative to transmural myocardial perfusion in anesthetized dogs. Circulation 1980;62:1266.

9. Weintraub WS, Hattori S, Agarwal JB, Bodenheimer MM Banka VS, Helfant RH. Relationship between myocardial blood flow and contraction by myocardial layer in the canine left ventricle during ischemia. Circ Res 1981;48:430.

10. Pandian NG, Kerber RE. Two-dimensional echocardiography in experimental coronary stenosis. Sensitivity and specificity in detecting transient myocardial dyskinesis: comparison with sonomicrometers. Circulation 1982;66:597.

11. Buda AJ, Zotz RJ. Serial assessment of circumferential regional left ventricular function following complete coronary occlusion. AM HEART J 1986;112;447.

12. Buda AJ, Zotz RJ, Pace DP, Krause LC, Turla M. Immediate rebound followed by deterioration of regional left ventricular function with coronary reperfusion. J Am Coll Cardiol $1986 ; 8: 333$.

13. Wyatt HL, Meerbaum S, Heng MK, Tir J, Gueret P, Corday E. Experimental evaluation of the extent of myocardial dysenergy and infarct size by two-dimensional echocardiography. Circulation 1981;63:607.

14. Nieminen M, Parisi A, O'Boyle JE, Folland ED, Khuri S, Kloner RA. Serial evaluation of myocardial thickening and thinning in acute experimental infarction: identification and quantification using two-dimensional echocardiography. Circulation 1981;66:174.

15. Pandian N, Koyanagi S, Skorton D, Collins C, Marcus M, Kerber R. Relations between two-dimensional echocardiographic wall thickening abnormalities, myocardial infarct size and coronary risk area in normal and hypertrophied myocardium in dogs. Am J Cardiol 1983;52:1318.

16. Gillam LD, Franklin TD, Foale RA, Wiske PS, Guyer DE, Hogan RD, Weyman AE. The natural history of regional wall motion in the acutely infarcted canine ventricle. J Am Coll Cardiol 1986;7:1325.

17. Gallagher KP, Osakada G, Matsuzaki M, Kemper WS, Ross Jr J. Myocardial flow and function with critical coronary stenosis in exercising dogs. Am J Physiol 1982;243:H698.

18. Gallagher KP, Matsuzaki M, Osakada G, Kemper WA, Ross J. Effect of exercise on the relationship between myocardial blood flow and systolic wall thickening in dogs with acute coronary stenosis. Circ Res 1983;52:716.

19. Gallagher KP, Kumada T, Battler A, Kemper WS, Ross Jr J. Isoproterenol-induced myocardial dysfunction in dogs with coronary stenosis. Am J Physiol 1982;242:H260.

20. Vatner SF, McRitchie RJ, Maroko PR, Patrick TA, Braunwald E. Effects of catecholamines, exercise and nitroglycerin on the normal and ischemic myocardium in conscious dogs. $J$ Clin Invest 1974;54:563.

21. Mason JR, Päläćc RT, F̂reeman ML, Virupannavar S, Loeb HS, Kaplan E, Gunnar RM. Thallium scintigraphy during dobutamine infusion: nonexercise-dependent screening test for coronary disease. AM HEART J 1984;70:481

22. Berthe C, Pierard LA, Hiernaux M, Trotteur G, Lempereur $P$, Carlier J, Kulbertus HE. Predicting the extent and 
location of coronary artery disease in acute myocardial infarction by echocardiography during dobutamine infusion. Am J Cardiol 1986;58:1167.

23. Rabinovitch MA, Kalff V, Chan W, Schork A, Gross MD, Vogel RA, Thrall JH, Pitt B. The effect of dobutamine on exercise performance in patients with symptomatic ischemic heart disease. AM HEART J 1984;107:81.

24. Myers JH, Stirling MC, Choy M, Buda AJ, Gallagher KP. Direct measurement of inner and outer wall thickening dynamics with epicardial echocardiography. Circulation $1986 ; 74: 164$.

25. Lew WYN, Chen Z, Guth B, Covell JW. Mechanisms of augmented segment shortening in nonischemic areas during acute ischemia of the canine left ventricle. Circ Res 1985; 56:351.

26. Flaherty JT, Weisfeldt ML, Bulkley JBH, Gardner TJ, Gott
VL, Jacobus WE. Mechanisms of ischemic myocardial cell damage assessed by phosphorous-31 nuclear magnetic resonance. Circulation 1982;65:561.

27. Gallagher KP, Buda AJ, Pace D, Gerren RA, Shlafer M. Failure of superoxide dismutase and catalase to alter size of infarct in conscious dogs after 3 hours of occlusion followed by reperfusion. Circulation 1986;73:1065.

28. Distante A, Rovai D, Picano E, Moscarelli E, Morales MA, Palambo C, L'A bhate A. Transient changes in left ventricular mechanics during attacks of Prinzmetal's angina: a twodimensional echocardiographic study. AM HEART J 1984; 108:440.

29. Maseri A, L'Abbate A, Barold G, Chierchia S, Marzilli M, Ballestra AM, Severi S, Parodi O, Biagini, Distante A, Pesola A. Coronary vasospasm us a possible cause of myocardial infarction. N Engl J Med 1978;299:1271.

\section{BOUND VOLUMES AVAILABLE TO SUBSCRIBERS}

Bound volumes of the AMERICAN HEART IOURNAL are available to subseribers (only) for the 1988 issues from the Publisher at a cost of $\$ 47.00$ ( $\$ 60.00$ international) for Vol. 115 (January-June) and Vol. 116 (July-December). Shipping charges are included. Each bound volume contains a subject and author index and all advertising is removed. Copies are shipped within 60 days after publication of the last issuc in the volume. The binding is durable buckram with the journal name, volume number, and year stamped in gold on the spine. Payment must accompany all orders. Contact The C. V. Mosby Company, Circulation Department, 11830 Westline Industrial Drive, St. Louis, Missouri 63146, USA; phone (800) 325-4177, ext. 351.

Subseriptions must be in force to qualify. Bound volumes are not available in place of a regular Journal subscription. 